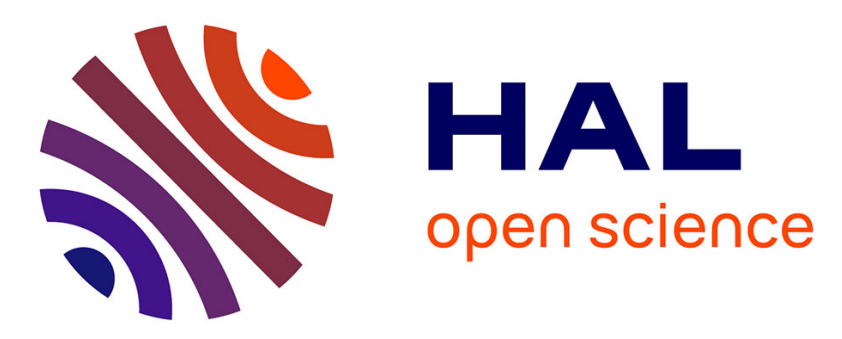

\title{
MuLoG: A Generic Variance-Stabilization Approach for Speckle Reduction in SAR Interferometry and SAR Polarimetry
}

Charles-Alban Deledalle, Loïc Denis, Florence Tupin

\section{- To cite this version:}

Charles-Alban Deledalle, Loïc Denis, Florence Tupin. MuLoG: A Generic Variance-Stabilization Approach for Speckle Reduction in SAR Interferometry and SAR Polarimetry. IGARSS 2018 - 2018 IEEE International Geoscience and Remote Sensing Symposium, Jul 2018, Valencia, France. pp.5816-5819, 10.1109/IGARSS.2018.8518346 。 ujm-03114598

\section{HAL Id: ujm-03114598}

\section{https://hal-ujm.archives-ouvertes.fr/ujm-03114598}

Submitted on 19 Jan 2021

HAL is a multi-disciplinary open access archive for the deposit and dissemination of scientific research documents, whether they are published or not. The documents may come from teaching and research institutions in France or abroad, or from public or private research centers.
L'archive ouverte pluridisciplinaire HAL, est destinée au dépôt et à la diffusion de documents scientifiques de niveau recherche, publiés ou non, émanant des établissements d'enseignement et de recherche français ou étrangers, des laboratoires publics ou privés. 


\title{
MULOG: A GENERIC VARIANCE-STABILIZATION APPROACH FOR SPECKLE REDUCTION IN SAR INTERFEROMETRY AND SAR POLARIMETRY
}

\author{
Charles-Alban Deledalle ${ }^{1}$,Lö̈ Denis ${ }^{2}$, Florence Tupin ${ }^{3}$ \\ ${ }^{1}$ IMB, CNRS, Univ Bordeaux, Bordeaux INP, F-33405 Talence, France \\ ${ }^{2}$ Univ Lyon, UJM- Télécom Saint-Etienne, CNRS, Institut d'Optique Graduate School, \\ Laboratoire Hubert Curien UMR 5516, F-42023, SAINT-ETIENNE, France \\ ${ }^{3}$ LTCI, Télécom ParisTech, Université Paris Saclay, Paris, France
}

\begin{abstract}
Speckle reduction is a long-standing topic in SAR data processing. Continuous progress made in the field of image denoising fuels the development of methods dedicated to speckle in SAR images. Adaptation of a denoising technique to the specific statistical nature of speckle presents variable levels of difficulty. It is well known that the logarithm transform maps the intrinsically multiplicative speckle into an additive and stationary component, thereby paving the way to the application of general-purpose image denoising methods to SAR intensity images. Multi-channel SAR images such as obtained in interferometric (InSAR) or polarimetric (PolSAR) configurations are much more challenging. This paper describes MuLoG, a generic approach for mapping a multi-channel SAR image into real-valued images with an additive speckle component that has a variance approximately constant. With this approach, general-purpose image denoising algorithms can be readily applied to restore InSAR or PolSAR data. In particular, we show how recent denoising methods based on deep convolutional neural networks lead to state-of-the art results when embedded with MuLoG framework.
\end{abstract}

Index Terms - SAR tomography, structural information, spatial regularization

\section{INTRODUCTION}

Most SAR satellites now offer not only an image of backscattered echoes but also polarimetric information and, by combining data from different satellites or different passes, interferometric information. Despite the efforts put during the development of Earth observation satellites to achieve a high spatial resolution, polarimetric capability and small temporal revisit times (to minimize temporal decorrelation in interferometric analyses), SAR images suffer from an intrinsic limitation due to speckle phenomenon. Coherent combination

This project has been funded in part by ANR (the French National Research Agency) and DGA (Direction Générale de l'Armement) under ALYS project ANR-15-ASTR-0002 of several echoes back-scattered by elements located within the same resolution cell lead to constructive or destructive summations. The resulting complex amplitude suffers from strong fluctuations known as speckle. To exploit SAR satellites at their full potential, it is therefore crucial to apply image processing methods that reduce speckle while preserving at best the spatial, polarimetric and interferometric information.

The field of speckle reduction methods is rich of several decades of development. Several families of image processing techniques form the core of these methods. Neighborhood filters such as Lee's sigma filter [1,2] consider neighbor pixels whose values are close enough to the value of the pixel under consideration. Markov random fields model that some local pixel configurations (corresponding to flat areas or smooth edges) are more frequent than others, see for example $[3,4,5]$. Wavelets transforms are used to better separate useful signal from speckle fluctuations, the signal of interest is concentrated in a few large coefficients after a wavelet transform, see [6, 7]. Patch-based methods identify similar pixels by comparing small windows (so-called patches), filtering is then performed by adaptively selecting and combining the most relevant pixel values, see $[8,9,10,11]$ and the review [12].

Speckle reduction is much simpler in the case of singlechannel intensity images. Speckle fluctuations, multiplicative by nature, can be turned into additive and homoscedastic (i.e., with a stationary variance) by applying a logarithm transform. Speckle reduction then resembles Gaussian denoising and standard image denoising algorithms can be applied. The case of multi-channel images is much more challenging. Speckle fluctuations between the channels are indeed correlated and therefore require a joint processing.

This paper illustrates how MuLoG, a generic approach that we recently introduced [13], can alleviate the difficulty of applying state-of-the-art image denoising techniques to the case of speckle reduction in multi-channel SAR images.

The structure of the paper is the following: we first recall the speckle distribution for multi-channel SAR images, 
describe how speckle in multi-channel images can be transformed into an additive component with stabilized variance, then we present the general framework of MuLoG. Finally, we illustrate the performance of MuLoG for speckle reduction in PolSAR and InSAR images.

\section{SPECKLE STATISTICS IN MULTI-CHANNEL SAR IMAGES}

The diffusion vector $\vec{k} \in \mathbb{C}^{D}$ at a given pixel of a $D$-channel SAR image can be statistically modeled, under Goodman's fully developed speckle, as a random realization of Gaussian process:

$$
\mathrm{p}(\vec{k} \mid \boldsymbol{\Sigma})=\frac{1}{\pi^{D}|\boldsymbol{\Sigma}|} \exp \left(-\vec{k}^{\dagger} \boldsymbol{\Sigma}^{-1} \vec{k}\right)
$$

where $\boldsymbol{\Sigma}=\mathbb{E}\left\{\vec{k} \vec{k}^{\dagger}\right\}$ is a $D \times D$ complex covariance matrix with $\mathbb{E}$ the mathematical expectation, ${ }^{\dagger}$ indicates the Hermitian transpose and $|\boldsymbol{\Sigma}|$ stands for the determinant of matrix $\boldsymbol{\Sigma}$. Diagonal elements of $\boldsymbol{\Sigma}$ correspond to the radar cross-section of scatterers in each channel. Off-diagonal elements define the complex correlation between channels, and depending on the SAR configuration (InSAR, PolSAR, PolInSAR), convey interferometric and/or polarimetric information. To perform interferometric or polarimetric analyses, it is thus necessary to estimate $\boldsymbol{\Sigma}$. The maximum likelihood estimator corresponds to the sample covariance matrix $\boldsymbol{C}$ defined by:

$$
\boldsymbol{C}=\frac{1}{L} \sum_{t=1}^{L} \vec{k}_{t} \cdot \vec{k}_{t}^{\dagger}
$$

When $L \geq D$, the sample covariance matrix is distributed according to a Wishart distribution:

$$
p_{\boldsymbol{C}}(\boldsymbol{C} \mid \boldsymbol{\Sigma})=\frac{L^{L D}|\boldsymbol{C}|^{L-D}}{\Gamma_{D}(L)|\boldsymbol{\Sigma}|^{L}} \exp \left(-L \operatorname{tr}\left(\boldsymbol{\Sigma}^{-1} \boldsymbol{C}\right)\right)
$$

where $\operatorname{tr}$ stands for the matrix trace and $\Gamma$ is the multivariate gamma function. Fluctuations of the sample covariance matrix $C$ around the true covariance $\Sigma$ depend on $\Sigma$. In particular, the variance $\operatorname{Var}[\operatorname{tr}(C)]$ is equal to $\frac{1}{L} \operatorname{tr}\left(\boldsymbol{\Sigma}^{2}\right)$, see [14]. Speckle fluctuations in the covariance matrix $\boldsymbol{C}$ are thus signal-dependent, see figure 1.

\section{VARIANCE STABILIZATION VIA MATRIX LOGARITHM}

Speckle fluctuations can be made almost signal-independent by the matrix logarithm transform:

$$
\boldsymbol{C} \mapsto \tilde{\boldsymbol{C}}=\log \boldsymbol{C}=\mathbf{E} \operatorname{diag}(\tilde{\boldsymbol{\Lambda}}) \mathbf{E}^{-1},
$$

where $\tilde{\boldsymbol{\Lambda}}_{i}=\log \boldsymbol{\Lambda}_{i}, \mathbf{E} \in \mathbb{C}^{D \times D}$ is the matrix whose column vectors are eigenvectors (with unit norm) of $\boldsymbol{C}, \boldsymbol{\Lambda} \in \mathbb{R}_{+}^{D}$
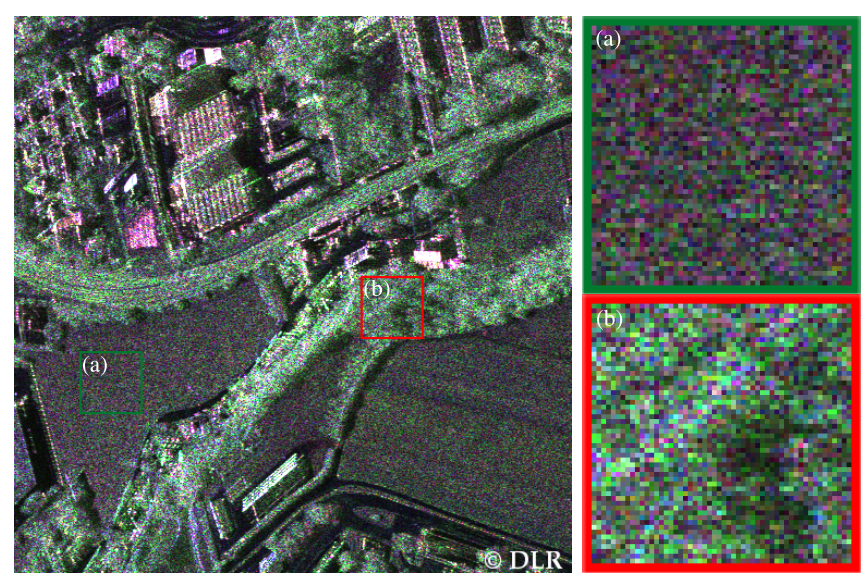

Fig. 1. Illustration of the non-stationarity of speckle in a polarimetric image (F-SAR airborne image CDLR): regions (a) and (b) display very different variances because the radiometry and polarimetric behavior differ in these two areas.

is the vector of corresponding eigenvalues, such that $C=$ $\mathbf{E} \operatorname{diag}(\boldsymbol{\Lambda}) \mathbf{E}^{-1}$, and $\tilde{\boldsymbol{\Lambda}} \in \mathbb{R}^{D}$. Its inverse transform is the matrix exponential defined similarly. Log-transformed covariance matrices $\tilde{C}$ are distributed according to a WishartFisher-Tippett distribution [13]:

$$
p_{\tilde{\boldsymbol{C}}}(\tilde{\boldsymbol{C}} \mid \tilde{\boldsymbol{\Sigma}})=\kappa e^{L \operatorname{tr}(\tilde{\boldsymbol{C}}-\tilde{\boldsymbol{\Sigma}})} \exp \left(-L \operatorname{tr}\left(e^{\tilde{\boldsymbol{C}}} e^{-\tilde{\boldsymbol{\Sigma}}}\right)\right)
$$

with $\kappa$ a scalar that depends only on $D, L$ and $\tilde{C}$ and that will be irrelevant when estimating $\tilde{\boldsymbol{\Sigma}}$ from a given $\tilde{\boldsymbol{C}}$. This distribution is the multi-channel generalization of the wellknown Fisher-Tippett distribution of log-transformed intensity images corrupted by speckle. The first two moments of the trace of $\tilde{C}$ are known in closed form [15]:

$$
\begin{aligned}
& \mathbb{E}[\operatorname{tr} \tilde{\boldsymbol{C}}]=\operatorname{tr} \tilde{\boldsymbol{\Sigma}}+\sum_{i=1}^{D} \Psi(0, L-i+1)-D \log L, \\
& \text { and } \operatorname{Var}[\operatorname{tr} \tilde{\boldsymbol{C}}]=\sum_{i=1}^{D} \Psi(1, L-i+1),
\end{aligned}
$$

which shows that, (i) like in the case of a single-channel intensity image, a bias is present when averaging log-transformed data, and (ii) the variance (of the trace) is signal-independent (i.e., independent from $\boldsymbol{\Sigma}$ ). Numerical experiments show that not only the variance of the trace is stabilized, but the variance of each term of the covariance matrix $C$ is approximately signal-independent. The matrix logarithm thus offers a way to extend Gaussian denoisers to speckle reduction in multichannel SAR images.

\section{MULOG GENERIC FRAMEWORK}

Figure 2 summarizes the approach followed by MuLoG to reduce speckle noise in an interferometric or polarimetric SAR 


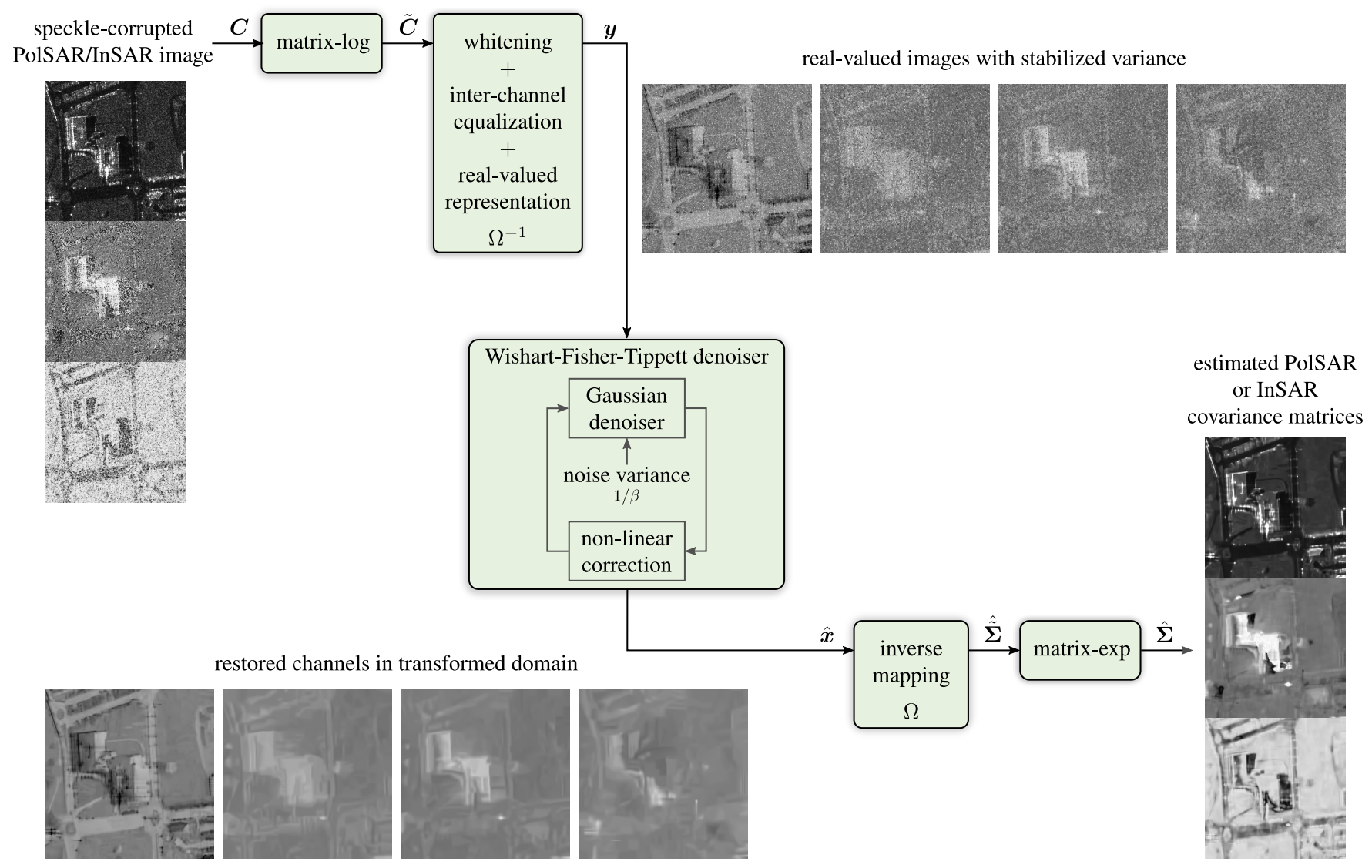

Fig. 2. MuLoG generic approach to speckle reduction in multi-channel SAR data. Images illustrate the case of SAR interferometry (RAMSES airborne sensor (C)ONERA), see also figure 3.

image. The speckle-corrupted input image (an InSAR image in the illustration) is transformed first by the matrix logarithm transformed introduced in equation (4), then by an affine transform that extracts real-valued channels (4 channels in the case of InSAR) where the information of interest is decorrelated and the speckle fluctuations are stationary and equalized. By decorrelating the channels, the subsequent Gaussian denoising steps can be applied separately on each channel. This is the core of the denoising process represented by the box "Wishart-Fisher-Tippett denoiser". Gaussian denoising steps are alternated with a non-linear correction applied jointly on all channels in order to account for the specific distribution of multi-channel SAR data that underwent a matrix-logarithm transform, as defined in equation (5). As can be observed on figure 2, at the end of the WishartFisher-Tippett denoising, the channels have almost no residual fluctuations. The final step of MuLoG is to recombine all channels and map them back to the original domain with a matrix exponential. A more detailed description of each step and their implementation is available in the journal paper [13] and the open-source implementation https://www . math.u-bordeaux.fr/ cdeledal/mulog.php.

\section{APPLICATION TO POLSAR AND INSAR}

MuLoG is a generic approach in the sense that (i) it applies to mono or multi-channel images (intensity, interferometric, polarimetric, PolInSAR, multi-baseline images), and (ii) the Gaussian denoiser embedded in the Wishart-Fisher-Tippett denoiser (central block in figure 2) can be selected among various state-of-the-art denoising algorithms in standard image processing literature. We illustrate this genericity with two speckle-reduction results. Figure 3 illustrates the capability of MuLoG to restore interferometric information. An airborne image (ONERA's RAMSES sensor) is processed with MuLoG and the well-established BM3D denoising algorithm [16]. Figure 4 presents a restoration result of polarimetric information on an airborne image acquired by the DLR's ESAR sensor. The Gaussian denoiser used is a very recent algorithm based on a convolutional neural network (CNN) [17].

\section{CONCLUSION}

This paper described MuLoG, a generic and open-source method for speckle reduction in SAR interferometry or SAR polarimetry. A distinctive feature of MuLoG is its ability to 

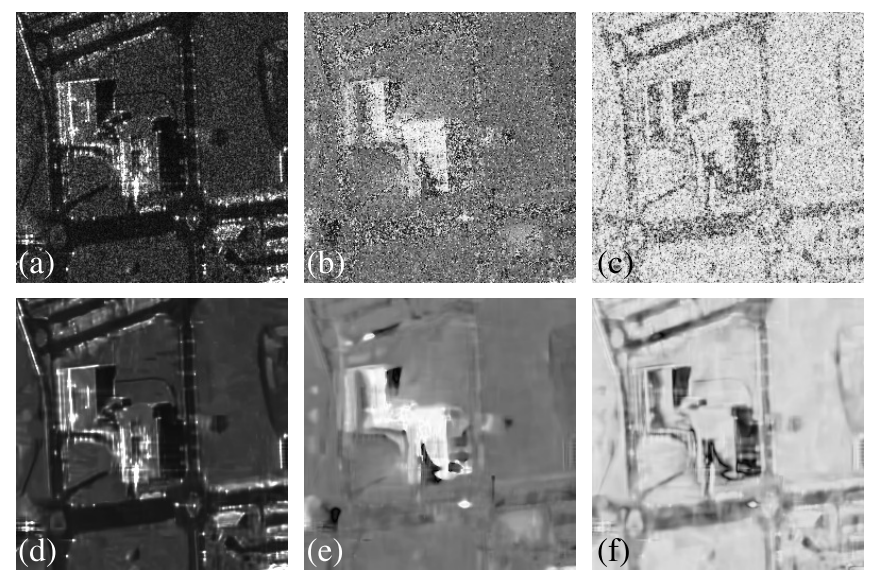

Fig. 3. Restoration of an InSAR image with MuLoG. First row: original speckle corrupted image (airborne image of Toulouse, France by RAMSES (c)ONERA) (a) intensity, (b) interferometric phase, (c) coherence; second row: restored image with MuLoG and BM3D Gaussian denoiser (d) intensity, (e) interferometric phase, (f) coherence.

include almost any standard Gaussian denoising algorithm. It therefore provides a simple and natural way to apply most recent denoising methods, in particular those based on deep learning. Moreover, by visually comparing the results obtained when using different denoising algorithms, most of the method-specific artifacts can be discarded.

\section{REFERENCES}

[1] J.S. Lee, "Digital noise smoothing and the sigma filter," Comput. Graph. Image Process, vol. 24, pp. 255-269, 1983.

[2] J-S Lee, T Ainsworth, Y Wang, and K-S Chen, "Polarimetric SAR speckle filtering and the extended sigma filter," IEEE Trans. Geosci. Remote Sens., vol. 53, no. 3, 2015.

[3] L Denis, F Tupin, J Darbon, and M Sigelle, "SAR image regularization with fast approximate discrete minimization," IEEE Trans. Image Process., vol. 18, no. 7, pp. 1588-1600, 2009.

[4] J Bioucas-Dias and M Figueiredo, "Multiplicative noise removal using variable splitting and constrained optimization," IEEE Trans. Image Process., vol. 19, no. 7, 2010.

[5] G Steidl and T Teuber, "Removing multiplicative noise by Douglas-Rachford splitting methods," J. Math. Imag. Vision, vol. 36, no. 2, pp. 168-184, 2010.

[6] H Xie, L Pierce, and F Ulaby, "SAR speckle reduction using wavelet denoising and Markov random field modeling," IEEE Trans. Geosci. Remote Sens., vol. 40, no. 10, pp. 2196-2212, 2002.

[7] A Achim, P Tsakalides, and A Bezerianos, "SAR image denoising via Bayesian wavelet shrinkage based on heavy-tailed modeling," IEEE Trans. Geosci. Remote Sens., vol. 41, no. 8, pp. 1773-1784, 2003.
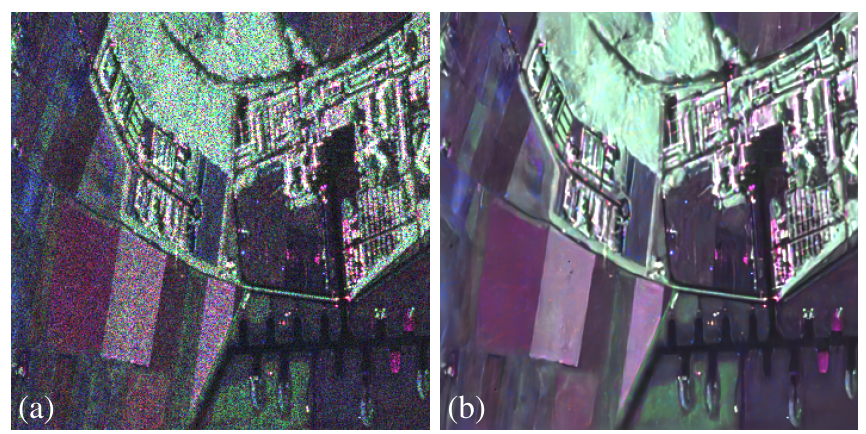

Fig. 4. Restoration of a polarimetric image with MuLoG: (a) Pauli color representation of the original image (singlelook airborne image of Oberpfaffenhofen, Germany by ESAR (CDLR), (b) restoration with MuLoG and a Gaussian denoiser based on deep learning (CNN proposed in [17]).

[8] C Deledalle, L Denis, and F Tupin, "Iterative weighted maximum likelihood denoising with probabilistic patch-based weights," IEEE Trans. Image Process., vol. 18, no. 12, pp. 2661-2672, 2009.

[9] J Chen, Y Chen, W An, Y Cui, and J Yang, "Nonlocal filtering for polarimetric SAR data: A pretest approach," IEEE Trans. Geosci. Remote Sens., vol. 49, no. 5, pp. 1744-1754, 2011.

[10] S Parrilli, M Poderico, C Angelino, and L Verdoliva, "A nonlocal sar image denoising algorithm based on llmmse wavelet shrinkage," IEEE Trans. Geosci. Remote Sens., vol. 50, no. 2, pp. 606-616, 2012.

[11] C Deledalle, L Denis, F Tupin, A Reigber, and M Jäger, "NLSAR: A unified nonlocal framework for resolution-preserving (Pol)(In) SAR denoising," IEEE Trans. Geosci. Remote Sens., vol. 53 , no. $4,2015$.

[12] C Deledalle, L Denis, G Poggi, F Tupin, and L Verdoliva, "Exploiting patch similarity for sar image processing: the nonlocal paradigm,” IEEE Signal Process. Mag., vol. 31, no. 4, 2014.

[13] C Deledalle, L Denis, S Tabti, and F Tupin, "MuLoG, or How to apply Gaussian denoisers to multi-channel SAR speckle reduction?," IEEE Trans. Image Process., 2017.

[14] NR Goodman, "The distribution of the determinant of a complex Wishart distributed matrix," The Annals of mathematical statistics, vol. 34, no. 1, pp. 178-180, 1963.

[15] S N Anfinsen, A Doulgeris, and T Eltoft, "Estimation of the equivalent number of looks in polarimetric synthetic aperture radar imagery," IEEE Trans. Geosci. Remote Sens., vol. 47, no. 11, pp. 3795-3809, 2009.

[16] K Dabov, A Foi, V Katkovnik, and K Egiazarian, "Image denoising by sparse 3-D transform-domain collaborative filtering," IEEE Trans. Image Process., vol. 16, no. 8, pp. 20802095, 2007.

[17] K Zhang, W Zuo, S Gu, and L Zhang, "Learning Deep CNN Denoiser Prior for Image Restoration,” arXiv:1704.03264, 2017. 